\title{
The acquisition queue
}

\author{
D. Denteneer · J.S.H. van Leeuwaarden • I.J.B.F. Adan
}

Received: 19 September 2006 / Revised: 22 May 2007 / Published online: 15 June 2007

(C) Springer Science+Business Media, LLC 2007

\begin{abstract}
We propose a new queueing model named the acquisition queue. It differs from conventional queueing models in that the server not only serves customers, but also performs acquisition of new customers. The server has to divide its energy between both activities. The number of newly acquired customers is uncertain, and the effect of the server's acquisition efforts can only be seen after some fixed time period $\delta$ (delay).

The acquisition queue constitutes a $(\delta+1)$-dimensional Markov chain. The limiting queue length distribution is derived in terms of its probability generating function, and an exact expression for the mean queue length is given. For large values of $\delta$ the numerical procedures needed for calculating the mean queue length become computationally cumbersome. We therefore complement the exact expression with a fluid approximation.

One of the key features of the acquisition queue is that the server performs more acquisition when the queue is small. Together with the delay, this causes the queue length process to show a strongly cyclic behavior. We propose and investigate several ways of planning the acquisition efforts. In particular, we propose an acquisition scheme that is designed
\end{abstract}

This research was financially supported by the European Network of Excellence Euro-NGI. The work of the second author was supported in part by a TALENT grant from the Netherlands Organisation for Scientific Research (NWO).

D. Denteneer

Philips Research, 5656 AA, Eindhoven, The Netherlands

J.S.H. van Leeuwaarden ( $\varangle) \cdot$ I.J.B.F. Adan

Department of Mathematics and Computer Science,

EURANDOM and Eindhoven University of Technology,

P.O. Box 513, $5600 \mathrm{MB}$, Eindhoven, The Netherlands

e-mail: j.s.h.v.leeuwaarden@tue.nl specifically to reduce the cyclic behavior of the queue length process.

Keywords Acquisition queue · Delay · Higher-dimensional Markov chain · Queueing theory . Discrete-time · Generating function approach · Fluid approximation $\cdot$ Scheduling $\cdot$ Cyclic behavior

Mathematics Subject Classification (2000) 60K25 . $68 \mathrm{M} 20 \cdot 90 \mathrm{~B} 22 \cdot 34 \mathrm{H} 05 \cdot 60 \mathrm{~J} 20$

\section{Introduction}

In conventional queueing systems customers arrive at a service entity according to some renewal process that is independent of the service process. In this paper we introduce a model that requires the server to perform itself the acquisition of customers. The server has to divide its energy between serving customers presently waiting and performing acquisition of new customers. The number of newly acquired customers is uncertain, and the effect of the server's acquisition efforts can only be seen after some fixed period of time.

We divide time into slots of equal length, and we assume that in each slot the server has $\eta$ units of energy to spend, where one unit of energy is needed to serve one customer. The server divides its energy according to $s$ units for serving customers, and $\alpha$ units for performing acquisition. Hence $\eta=s+\alpha$ with $\eta, s, \alpha$ non-negative integers. Let $X_{n}$ denote the number of customers waiting in the queue at the beginning of time slot $n$. We assume that the server does not waste energy, so when there are fewer than $s$ customers present at the beginning of a time slot $n$, the server will use $\alpha_{n}=\alpha+\max \left\{0, s-X_{n}\right\}$ units of energy for acquisition. 
Let $A$ denote the random variable that represents the number of customers acquired with one unit of energy spent on acquisition. We assume that customers acquired due to an acquisition effort in slot $n$ arrive at the queue at the beginning of slot $n+\delta+1$ (with $\delta$ some non-negative integer). The queue length process can then be described as $X_{0}=0$ and

$X_{n+1}=\left(X_{n}-s\right)^{+}+\sum_{k=1}^{\alpha_{n-\delta}} A_{n, k}, \quad n=0,1, \ldots$,

where $x^{+}=\max \{0, x\}$ and the $A_{n, k}$ are random variables i.i.d. according to $A$. We refer to the queueing model defined by (1) as the acquisition queue.

\subsection{Motivation}

The acquisition queue is motivated from data transfer in cable networks organized via a request-grant procedure. In these networks, a user should first send a request to some central server, and once this request gets granted, the user waits in the data queue until the data of his actual message gets transmitted. The central server schedules the available network capacity among the processes of handling requests and transmitting data.

In cable networks, there is typically a substantial transmission delay, defined as the time required to transmit a signal from the user to the central server and vice versa. Due to this delay, scheduling decisions must be taken in advance so that they can be communicated to the users. Consequently, there is a time lag between granting a request and transmitting the data associated with the request. Therefore, one is naturally led to consider periodical scheduling, for which slots are grouped together into frames of consecutive slots, $\eta$ say. The nature of each slot in the frame is periodically determined by the central server and broadcast to all users.

Let $X_{n}$ now denote the size of the data queue at the beginning of frame $n$. Let $\delta$ represent the transmission delay such that a request made in frame $n$ can be scheduled at the earliest in frame $n+\delta+1$. Further assume that every frame, $\alpha$ slots are used for handling new requests. Then, the acquisition queue (1) serves as a model for the data queue. The quantity $\alpha_{n}$ can be interpreted as the number of slots in frame $n$ that are used for handling requests, and the sum in (1) equals the total number of new data packets for which transmission is granted. For more background on this application, we refer to $[2,3]$.

\subsection{Key features}

One of the key features of (1) is that there is some natural form of input balancing. When there is little work (i.e. $X_{n}<s$ ), more energy is spent on acquisition, which

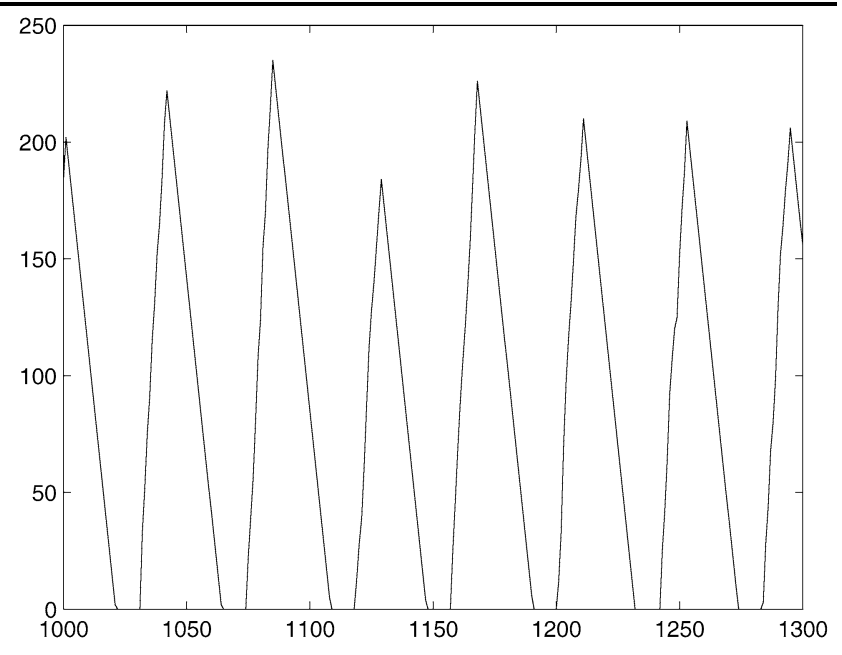

Fig. 1 Sample path of the process defined by (1), for $\eta=10, \delta=10$, $\alpha=0$, and $A$ Poisson distributed with $\mu_{A}=3$

is expected to result in more work in the future. Another key feature is the delay $\delta$ between the acquisition effort and the actual arrival of the acquired customers. This not only makes the analysis harder (note that $(1)$ is a $(\delta+1)$-dimensional Markov chain), but it might also corrupt the input balancing. Indeed, when newly acquired customers arrive at the queue, the situation might be totally different from that of $\delta+1$ slots ago. A third feature of (1) is the cyclic behavior. It is intuitively clear that the delay $\delta$ may result in a strongly correlated arrival process, and therefore in a cyclic behavior of the queue length process. Figure 1 displays a sample path for slot 1,000 until slot 1,300 for $\eta=10, \delta=10, \alpha=0$, and $A$ Poisson distributed with $\mu_{A}=3$.

We see in Fig. 1 that the sample path has settled on a cyclic pattern. Each cycle can be subdivided into three consecutive periods. First, there is a period of length $\delta+1$ in which the queue length is approximately zero. Then there is a period of length $\delta+1$ in which the queue length increases. Finally, in a third period, the queue is drained until it hits zero, upon which a new cycle starts. Figures 2, 3 display results for $\alpha=1,2$. The larger $\alpha$, the less restrictive the pattern. Figure 4 displays results for $\delta=0$, in which case the cyclic pattern is no longer there.

This cyclic behavior might have severe consequences for performance measures like the mean and variance of the queue length, and we aim at smoothing the arrival process and reducing the correlation of the arrival process by planning the server's acquisition efforts in an appropriate way.

\subsection{Acquisition planning}

The parameter $\alpha$ in $\alpha_{n}=\alpha+\left(s-X_{n}\right)^{+}$, see (1), can be interpreted as the amount of energy guaranteed for acquisition. In each slot at least $\alpha$ energy units are used for acquisition. Since the value of $\alpha$ is fixed, we refer to this type of acquisition planning as static acquisition planning. The value of 


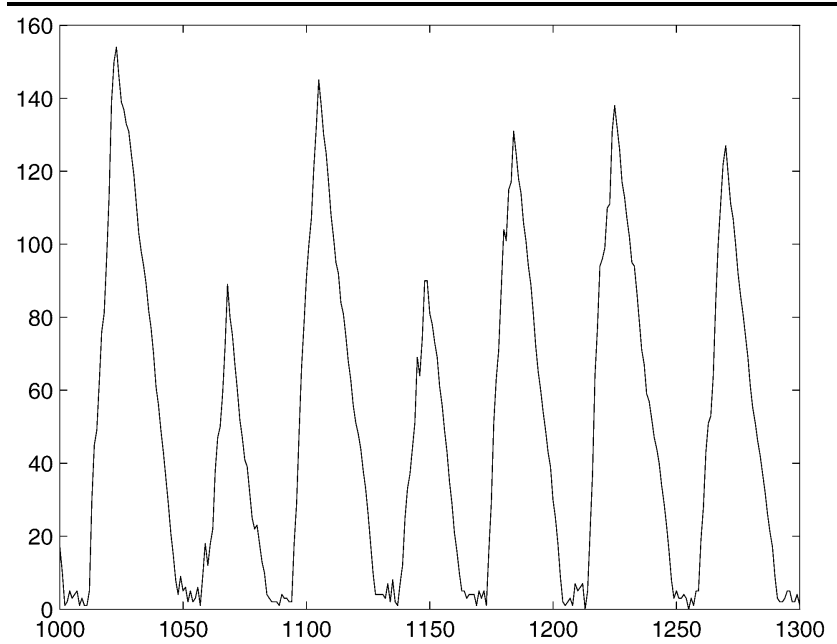

Fig. 2 Sample path of the process defined by (1), for $\eta=10, \delta=10$, $\alpha=1$, and $A$ Poisson distributed with $\mu_{A}=3$

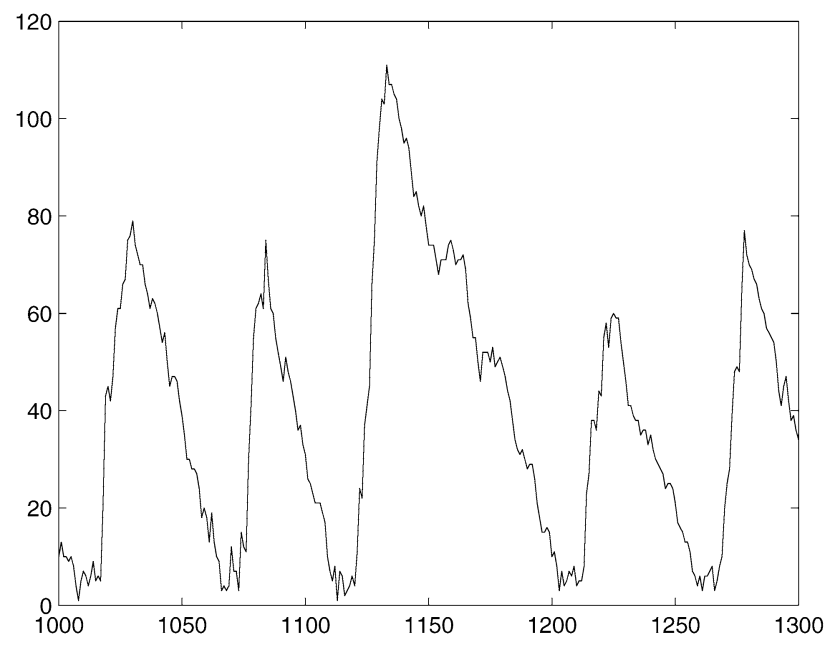

Fig. 3 Sample path of the process defined by (1), for $\eta=10, \delta=10$, $\alpha=2$, and $A$ Poisson distributed with $\mu_{A}=3$

$\alpha$ has an impact on the queue length process. The heuristic rationale is that a larger value of $\alpha$ makes the arrival process more stable, but less adaptive to the current queue length. That is, the larger $\alpha$, the less room, i.e. $\left(\eta-\alpha-X_{n}\right)^{+}$, there is for input balancing.

There are thus two, unfortunately conflicting, heuristics that guide a judicious choice of $\alpha$. On the one hand, setting $\alpha$ small ensures that customers are served as quickly as possible, and one would expect that this keeps the queue of waiting customers small. On the other hand, setting $\alpha$ large stabilizes the arrival process, which could possibly lead to smaller queue lengths. In choosing the right value of $\alpha$, one should strike the proper balance between these two considerations.

One could also consider a model in which the server is totally free to choose the acquisition effort in slot $n, \bar{\alpha}_{n}$ units

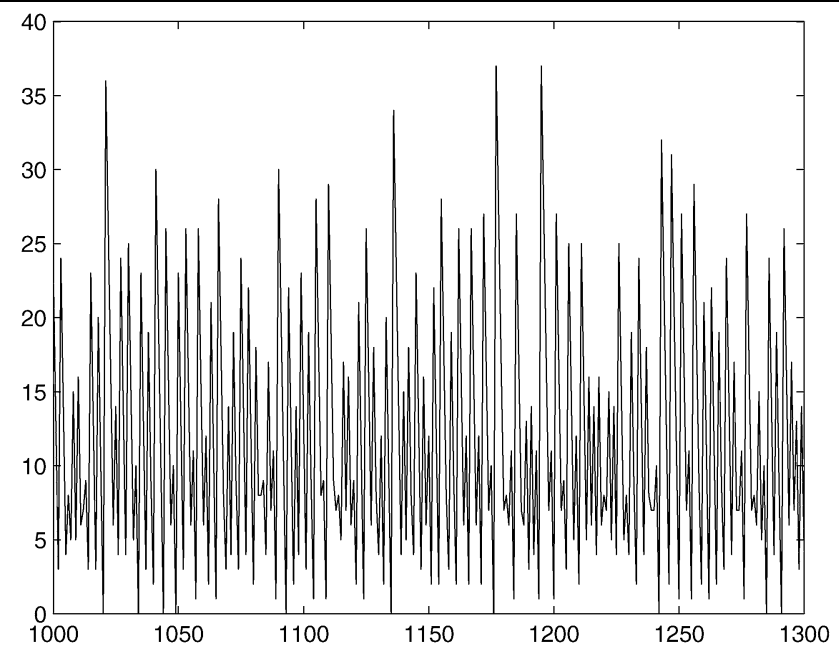

Fig. 4 Sample path of the process defined by (1), for $\eta=10, \delta=0$, $\alpha=0$, and $A$ Poisson distributed with $\mu_{A}=3$

say. This type of free acquisition planning then leads to the following modification of the acquisition queue

$\bar{X}_{n+1}=\left(\bar{X}_{n}-\left(\eta-\bar{\alpha}_{n}\right)\right)^{+}+\sum_{k=1}^{\bar{\alpha}_{n-\delta}} A_{n, k}$,

with $\bar{\alpha}_{n} \in\{0,1, \ldots, \eta\}$ for every $n$. We derive a scheme for choosing the value $\bar{\alpha}_{n}$ based on the queue length at the beginning of the slot and the acquisition effort in the previous $\delta$ slots (of which the result is still not known).

The goals of this paper are to derive the limiting queue length distribution for the acquisition queue (1) and to develop rules and heuristics for choosing $\alpha$ and $\bar{\alpha}_{n}$ in (2). We primarily aim at dealing with the cyclic behavior caused by the delay $\delta$.

\subsection{Outline of the paper}

For $\delta=0$, (1) defines a one-dimensional Markov chain, and the pgf of the limiting queue length distribution is obtained in van Leeuwaarden et al. [9]. For $\delta=1,2, \ldots$ the approach from [9] does not carry over. Yet, we are able to derive an exact solution, presented in Sect. 2. The solution, though, is rather complex, since it requires the determination of $s(s+1)^{\delta}$ boundary probabilities. We therefore derive in Sect. 3 an exact expression for the mean limiting queue length that is of more compact form and does allow for an intuitive interpretation, although it still contains terms that can only be calculated from the boundary probabilities. Since this calculation becomes prohibitively cumbersome for somewhat larger values of $\delta$, we develop in Sect. 4 an approximation for the mean queue length based on a fluid approximation. This approximation provides valuable information on the (cyclic) behavior of the delayed acquisition 
queue, which we use for developing rules as to choose an appropriate value for $\bar{\alpha}_{n}$ (free acquisition planning) in Sect. 5 . In Sect. 6, a simulation-based comparison is made between the performance of the various types of acquisition planning. We end in Sect. 7 with some conclusions.

\section{Exact solution}

Let $A(z)=\sum_{k=0}^{\infty} \mathbb{P}(A=k) z^{k}$ be the pgf of $A$ and define $a_{k}^{j}=\mathbb{P}\left(A_{1}+\cdots+A_{j}=k\right)$, where $A_{i}$ i.i.d. as $A$ for all $i$. Denote by $\mu_{A}$ and $\sigma_{A}^{2}$ the mean and variance of $A$. Let $M_{n}=$ $\left(s-X_{n}\right)^{+}$. From (1) it is clear that $\left\{\left(X_{n}, M_{n-1}, \ldots, M_{n-\delta}\right)\right\}$ is a Markov chain. The variables $M_{n-1}, \ldots, M_{n-\delta}$ constitute our memory, in the sense that these variables keep track of all acquisition efforts of which the outcome is still not known. We henceforth assume that this Markov chain is irreducible and aperiodic; for example, this holds when $\mathbb{P}(A=k)>0$ for all $k \geq 0$. The ergodicity condition is formulated in the following lemma.

Lemma 1 The Markov chain $\left\{\left(X_{n}, M_{n-1}, \ldots, M_{n-\delta}\right)\right\}$ is ergodic if

$\alpha \mu_{A}-s<0$.

Proof By partitioning the state space of the Markov chain $\left\{\left(X_{n}, M_{n-1}, \ldots, M_{n-\delta}\right)\right\}$ into levels $i$, where level $i$ is the subset of states for which the queue length is $i, i=0,1, \ldots$, it is readily seen that the probability transition matrix is an M/G/1-type stochastic matrix, see [10]. Hence, the ergodicity condition is the usual condition stating that the average drift should be negative, which in this case reduces to inequality (3). For an alternative proof of (3), based on Foster's criterion, the reader is referred to [2].

In the sequel we assume that (3) is satisfied. The Markov chain $\left\{\left(X_{n}, M_{n-1}, \ldots, M_{n-\delta}\right)\right\}$ then has a unique limiting distribution

$$
\begin{aligned}
& \pi\left(k, m_{1}, \ldots, m_{\delta}\right) \\
& \quad=\lim _{n \rightarrow \infty} \mathbb{P}\left(X_{n}=k, M_{n-1}=m_{1}, \ldots, M_{n-\delta}=m_{\delta}\right),
\end{aligned}
$$

where $k \geq 0$ and $m_{i} \in\{0, \ldots, s\}$ for all $i$. Let $X$ denote a random variable distributed according to the limiting queue length distribution

$\pi(k):=\mathbb{P}(X=k)=\lim _{n \rightarrow \infty} \mathbb{P}\left(X_{n}=k\right)$.

The probability generating function of $X$ is then given by

$$
G(z)=\sum_{m_{1}=0}^{s} \cdots \sum_{m_{\delta}=0}^{s} G_{m_{1}, \ldots, m_{\delta}}(z)
$$

where

$G_{m_{1}, \ldots, m_{\delta}}(z)=\sum_{k=0}^{\infty} \pi\left(k, m_{1}, \ldots, m_{\delta}\right) z^{k}$.

In the analysis below we make use of the normalization condition

$\sum_{k=0}^{s-1} \sum_{m_{1}=0}^{s} \cdots \sum_{m_{\delta}=0}^{s} \pi\left(k, m_{1}, \ldots, m_{\delta}\right)(s-k)=\frac{s-\alpha \mu_{A}}{1+\mu_{A}}$,

which can be explained as follows. The left-hand side of (8) clearly expresses the additional acquisition effort per slot. On average, the guaranteed acquisition effort brings per slot $\alpha \mu_{A}$ of new customers to the queue, each customer requiring one unit of energy. Hence, $\alpha \mu_{A}$ of the remaining $s$ energy units per slot are spent on serving these customers. This leaves $s-\alpha \mu_{A}$ energy units, of which only $\left(s-\alpha \mu_{A}\right) /\left(1+\mu_{A}\right)$ units can be spent on additional acquisition (since one unit of acquisition results on average in $\mu_{A}$ new customers).

\subsection{Case $\delta=0$}

We start from the balance equations (for $k=0,1, \ldots$ )

$\pi(k)=\sum_{k^{\prime}=s}^{k+s} \pi\left(k^{\prime}\right) a_{k-k^{\prime}+s}^{\alpha}+\sum_{k^{\prime}=0}^{s-1} \pi\left(k^{\prime}\right) a_{k}^{\alpha+s-k^{\prime}}$.

Multiplying both sides of (9) by $z^{k}$, summing over all values of $k$, and rearranging terms yields

$G(z)=\frac{A(z)^{\alpha} \sum_{k=0}^{s-1} \pi(k)\left(z^{s} A(z)^{s-k}-z^{k}\right)}{z^{s}-A(z)^{\alpha}}$.

Expression (10) is of indeterminate form, but the $s$ boundary probabilities $\pi(0), \ldots, \pi(s-1)$ can be determined by consideration of the zeros of the denominator in (10) that lie on or within the unit circle. The following lemma is taken from [1].

Lemma 2 If $\alpha \mu_{A}<s$ and $\mathbb{P}(A=0)>0$, it holds that $z^{s}-$ $A(z)^{\alpha}$ has $s$ zeros on or within the unit circle.

Denote the $s$ zeros of $z^{s}-A(z)^{\alpha}$ in $|z| \leq 1$ by $z_{0}=$ $1, z_{1}, \ldots, z_{s-1}$, which are assumed to be simple (and thus distinct). Since the function $G(z)$ is finite on and inside the unit circle, the numerator of the right-hand side of (10) needs to be zero for each of the $s$ zeros, i.e., the numerator should vanish at the exact points where the denominator of the right-hand side of (10) vanishes. For $z_{0}=1$, this is trivial, so Lemma 2 and (10) lead to $s-1$ (non-trivial) equations in terms of the $s$ boundary probabilities. The final equation follows from the normalization condition (8). 
If $z^{s}-A(z)^{\alpha}$ has zeros of multiplicity greater than two, sufficiently many equations are obtained from setting the derivatives (up to the multiplicity) of the numerator of the right-hand side of (10) equal to zero.

\subsection{Case $\delta=1$}

In this case we include one memory variable $M_{n-1}$ into our state description, and we distinguish between the balance equations for states $\left(k, m_{1}\right)$ with $m_{1}=0$

$\pi(k, 0)=\sum_{i=0}^{s} \sum_{k^{\prime}=s}^{k+s} \pi\left(k^{\prime}, i\right) a_{k-k^{\prime}+s}^{\alpha+i}$,

and with $m_{1} \in\{1, \ldots, s\}$

$\pi\left(k, m_{1}\right)=\sum_{i=0}^{s} \pi\left(s-m_{1}, i\right) a_{k}^{\alpha+i}$.

Multiplying both sides of (11) and (12) by $z^{k}$ and summing over all values of $k$ yields

$G_{0}(z)=z^{-s} \sum_{i=0}^{s}\left(G_{i}(z)-\sum_{k=0}^{s-1} \pi(k, i) z^{k}\right) A(z)^{\alpha+i}$,

and for $m_{1} \in\{1, \ldots, s\}$

$G_{m_{1}}(z)=\sum_{i=0}^{s} \pi\left(s-m_{1}, i\right) A(z)^{\alpha+i}$

Upon substituting (14) into (13) and rearranging terms we find

$G_{0}(z)$

$$
=\frac{A(z)^{\alpha}\left(\sum_{i=0}^{s} \sum_{k=0}^{s-1} \pi(k, i)\left(A(z)^{s+\alpha+i-k}-z^{k} A(z)^{i}\right)\right.}{z^{s}-A(z)^{\alpha}} .
$$

Hence, (14) and (15) still contain $s(s+1)$ boundary probabilities

$\pi\left(k, m_{1}\right), \quad k=0, \ldots, s-1, m_{1}=0, \ldots, s$,

which should be determined. We therefore match these unknowns by equally many equations: $s^{2}$ equations follow from (12), $s-1$ equations follow from Lemma 2 and (15), and a final equation is provided by the normalization condition (8).

\subsection{Case $\delta=2$}

We now include two memory variables $M_{n-1}$ and $M_{n-2}$ into our state description, but for the balance equations we only need to distinguish between the states $\left(k, m_{1}, m_{2}\right)$ with $m_{1}=0$ and $m_{1} \in\{1, \ldots, s\}$. We get

$\pi\left(k, 0, m_{2}\right)=\sum_{i=0}^{s} \sum_{k^{\prime}=s}^{k+s} \pi\left(k^{\prime}, m_{2}, i\right) a_{k-k^{\prime}+s}^{\alpha+i}$,

and for $m_{1} \in\{1, \ldots, s\}$

$\pi\left(k, m_{1}, m_{2}\right)=\sum_{i=0}^{s} \pi\left(s-m_{1}, m_{2}, i\right) a_{k}^{\alpha+i}$,

and so we obtain for $m_{2} \in\{0, \ldots, s\}$

$\begin{aligned} G_{0, m_{2}}(z)= & z^{-s} \sum_{i=0}^{s}\left(G_{m_{2}, i}(z)\right. \\ & \left.-\sum_{k=0}^{s-1} \pi\left(k, m_{2}, i\right) z^{k}\right) A(z)^{\alpha+i},\end{aligned}$

$G_{m_{1}, m_{2}}(z)=\sum_{i=0}^{s} \pi\left(s-m_{1}, m_{2}, i\right) A(z)^{\alpha+i}$,

$m_{1} \in\{1, \ldots, s\}$.

Upon rearranging terms in (18) for $m_{2}=0$ we get

$$
\begin{aligned}
& G_{0,0}(z) \\
& \quad=\frac{A(z)^{\alpha}\left(\sum_{i=1}^{s} G_{0, i}(z) A(z)^{i}-\sum_{i=0}^{s} \sum_{k=0}^{s-1} \pi(k, 0, i) z^{k} A(z)^{i}\right)}{z^{s}-A(z)^{\alpha}} .
\end{aligned}
$$

Equations (18-20) contain $s(s+1)^{2}$ boundary probabilities $\pi\left(k, m_{1}, m_{2}\right), \quad k \in\{0, \ldots, s-1\}, m_{1}, m_{2} \in\{0, \ldots, s\}$,

which can again be matched by equally many equations. In this case, $s^{2}(s+1)$ equations follow from (17) for $k \in$ $\{0, \ldots, s-1\}, m_{1} \in\{1, \ldots, s\}, m_{2} \in\{0, \ldots, s\}, s-1$ equations follow from Lemma 2 and (20) (using (18) and (19)), and one equation follows from the normalization condition (8). So we need an extra $s^{2}$ equations. For this, we consider the probabilities $\pi\left(k, 0, m_{2}\right), k \in\{0, \ldots, s-1\}$, $m_{2} \in\{1, \ldots, s\}$, see Fig. 5. Note that these probabilities can be expressed through (16) in terms of the probabilities $\pi\left(k^{\prime}, m_{2}, i\right), k^{\prime} \in\{s, \ldots, 2 s-1\}, m_{2} \in\{1, \ldots, s\}, i \in$ $\{0, \ldots, s\}$, only. Each of the latter probabilities can be written in terms of the boundary probabilities through (17), yielding $s^{2}$ equations.

\subsection{General case}

It might be clear from the analysis for $\delta=2$ that we can take a similar approach for $\delta=3,4, \ldots$ We start from the 


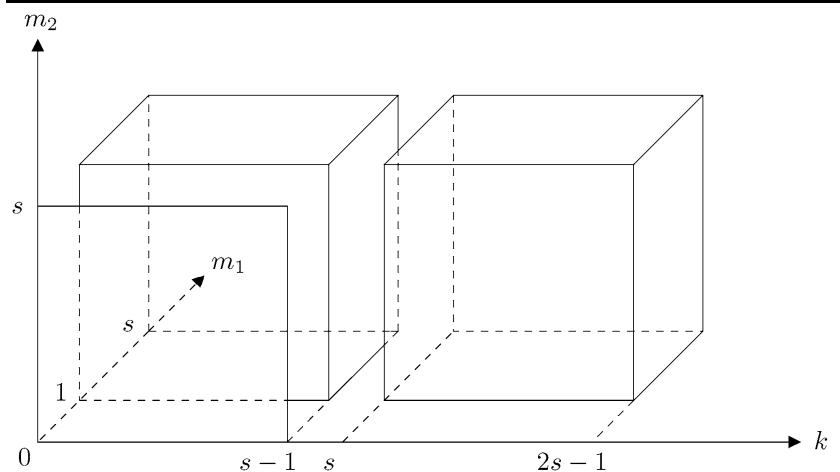

Fig. 5 The states corresponding to the boundary probabilities (21) and the additional probabilities $\pi\left(k^{\prime}, m_{2}, i\right), k^{\prime} \in\{s, \ldots, 2 s-1\}$, $m_{2} \in\{1, \ldots, s\}, i \in\{0, \ldots, s\}$

balance equations

$$
\begin{aligned}
\pi & \left(k, 0, m_{2}, \ldots, m_{\delta}\right) \\
= & \sum_{i=0}^{s} \sum_{k^{\prime}=s}^{k+s} \pi\left(k^{\prime}, m_{2}, \ldots, m_{\delta}, i\right) a_{k-k^{\prime}+s}^{\alpha+i},
\end{aligned}
$$

and for $m_{1} \in\{1, \ldots, s\}$

$$
\begin{aligned}
& \pi\left(k, m_{1}, m_{2}, \ldots, m_{\delta}\right) \\
& =\sum_{i=0}^{s} \pi\left(s-m_{1}, m_{2}, \ldots, m_{\delta}, i\right) a_{k}^{\alpha+i},
\end{aligned}
$$

and obtain

$$
\begin{aligned}
G_{0, m_{2}, \ldots, m_{\delta}}(z)= & z^{-s} \sum_{i=0}^{s}\left(G_{m_{2}, \ldots, m_{\delta}, i}(z)\right. \\
& \left.-\sum_{k=0}^{s-1} \pi\left(k, m_{2}, \ldots, m_{\delta}, i\right) z^{k}\right) A(z)^{\alpha+i},
\end{aligned}
$$

and for $m_{1} \in\{1, \ldots, s\}$

$G_{m_{1}, \ldots, m_{\delta}}(z)=\sum_{i=0}^{s} \pi\left(s-m_{1}, m_{2}, \ldots, m_{\delta}, i\right) A(z)^{\alpha+i}$.

For $m_{2}=m_{3}=\cdots=m_{\delta}=0$ we get from (24)

$$
\begin{aligned}
& G_{0, \ldots, 0}(z) \\
& =\frac{\sum_{i=1}^{s} G_{0, \ldots, 0, i}(z) A(z)^{\alpha+i}-\sum_{i=0}^{s} \sum_{k=0}^{s-1} \pi(k, 0, \ldots, 0, i) z^{k} A(z)^{\alpha+i}}{z^{s}-A(z)^{\alpha}} .
\end{aligned}
$$

We should then still determine the $s(s+1)^{\delta}$ boundary probabilities

$\pi\left(k, m_{1}, \ldots, m_{\delta}\right)$,

$$
k \in\{0, \ldots, s-1\}, m_{1}, \ldots, m_{\delta} \in\{0, \ldots, s\}
$$

and so we need equally many equations. Equation (23) immediately yields $s^{2}(s+1)^{\delta-1}$ equations, and we thus search for an extra $s(s+1)^{\delta-1}$ equations.

Consider (22) for $m_{2} \in\{1, \ldots, s\}$. The probabilities on the right-hand side of (22) can be written in terms of the boundary probabilities through (23), which yields $s^{2}(s+1)^{\delta-2}$ equations. Likewise, (22) for $m_{2}=0$ and $m_{3} \in$ $\{1, \ldots, s\}$ yields $s^{2}(s+1)^{\delta-3}$ equations, and we can repeat this trick all the way up to (22) for $m_{2}=\cdots=m_{\delta-1}=0$, $m_{\delta} \in\{1, \ldots, s\}$ (yielding $s^{2}$ equations). Altogether, this leads to

$s^{2}(s+1)^{\delta-2}+s^{2}(s+1)^{\delta-3}+\cdots+s^{2}$

equations. Together with the $s-1$ equations from Lemma 2 and (26), and the normalization condition (8), we then have exactly $s(s+1)^{\delta-1}$ equations.

A demonstration of the implementation of this analytical solution will be provided in Sect. 3 (see Table 1).

\section{Mean limiting queue length}

In principle, the mean of $X$ follows from evaluating the first derivative of (6) at $z=1$, but this does not lead to a particularly nice expression. Instead, we apply a method that was first used by Kingman [8], which is based on the manipulation of

$P_{n}=\left(X_{n}-s\right)^{+}, \quad M_{n}=\left(s-X_{n}\right)^{+}$.

For these variables we have

$$
X_{n}-s=P_{n}-M_{n}, \quad\left(X_{n}-s\right)^{2}=P_{n}^{2}+M_{n}^{2} .
$$

Let $M$ denote a random variable distributed according to the limiting distribution of $M_{n}$, and hence $M$ is equal in distribution to $(s-X)^{+}$. We then derive the following result.

Theorem 1 The mean limiting queue length in the acquisition queue defined by (1) can be expressed as

$$
\begin{aligned}
\mathbb{E} X= & \frac{\alpha \sigma_{A}^{2}}{2\left(s-\alpha \mu_{A}\right)}+\frac{\sigma_{A}^{2}}{2\left(1+\mu_{A}\right)}+\frac{s+\alpha \mu_{A}}{2} \\
& +\mathbb{E}\left(M^{2}\right) \frac{\mu_{A}^{2}-1}{2\left(s-\alpha \mu_{A}\right)}+\mathbb{E} R \frac{\mu_{A}}{s-\alpha \mu_{A}},
\end{aligned}
$$

where

$$
\mathbb{E} R=\lim _{n \rightarrow \infty} \mathbb{E}\left(P_{n} M_{n-\delta}\right)
$$

Proof Define

$$
S_{n-\delta}=\sum_{i=1}^{\alpha+M_{n-\delta}} A_{i}
$$


Since $X_{n}-s=P_{n}-M_{n}=X_{n+1}-S_{n-\delta}-M_{n}$, taking expectations at both sides, letting $n \rightarrow \infty$, and rearranging terms gives

$\mathbb{E} M=\frac{s-\alpha \mu_{A}}{1+\mu_{A}}$.

Next, using $P_{n}=X_{n+1}-S_{n-\delta}$ and (29) yields

$$
\begin{aligned}
\left(X_{n}-s\right)^{2} & =P_{n}^{2}+M_{n}^{2}=\left(X_{n+1}-S_{n-\delta}\right)^{2}+M_{n}^{2} \\
& =X_{n+1}^{2}-2 X_{n+1} S_{n-\delta}+S_{n-\delta}^{2}+M_{n}^{2} \\
& =X_{n+1}^{2}-2\left(P_{n}+S_{n-\delta}\right) S_{n-\delta}+S_{n-\delta}^{2}+M_{n}^{2} \\
& =X_{n+1}^{2}-2 P_{n} S_{n-\delta}-S_{n-\delta}^{2}+M_{n}^{2},
\end{aligned}
$$

i.e.,

$2 s X_{n}=s^{2}+X_{n}^{2}-X_{n+1}^{2}+2 P_{n} S_{n-\delta}+S_{n-\delta}^{2}-M_{n}^{2}$.

Furthermore, from (32),

$$
\begin{aligned}
\mathbb{E}\left(S_{n-\delta}^{2}\right)= & \left(\alpha+\mathbb{E}\left(M_{n-\delta}\right)\right) \sigma_{A}^{2} \\
& +\left(\alpha^{2}+2 \alpha \mathbb{E}\left(M_{n-\delta}\right)+\mathbb{E}\left(M_{n-\delta}^{2}\right)\right) \mu_{A}^{2},
\end{aligned}
$$

and

$$
\begin{aligned}
\mathbb{E}\left(P_{n} S_{n-\delta}\right) & =\alpha \mathbb{E}\left(P_{n}\right) \mu_{A}+\mathbb{E}\left(P_{n} M_{n-\delta}\right) \mu_{A} \\
& =\alpha \mathbb{E}\left(X_{n}-s+M_{n}\right) \mu_{A}+\mathbb{E}\left(P_{n} M_{n-\delta}\right) \mu_{A} .
\end{aligned}
$$

Taking expectations in (35), substituting (36) and (37), letting $n \rightarrow \infty$, and rearranging terms leads to

$$
\begin{aligned}
2 \mathbb{E} X & \left(s-\alpha \mu_{A}\right) \\
= & \left(s-\alpha \mu_{A}\right)^{2}+2 \alpha \mathbb{E} M\left(\mu_{A}+\mu_{A}^{2}\right)+(\alpha+\mathbb{E} M) \sigma_{A}^{2} \\
& +\mathbb{E}\left(M^{2}\right)\left(\mu_{A}^{2}-1\right)+2 \mu_{A} \mathbb{E} R,
\end{aligned}
$$

with $\mathbb{E} R$ as in (31). Finally, substituting (33) into (38) yields (30).

Expression (30) contains two unknown terms: a term $\mathbb{E}\left(M^{2}\right)$ that is related to the energy spent on acquisition, and a correlation term $\mathbb{E} R$. Both terms can be expressed in terms of the boundary probabilities. One readily sees that

$$
\mathbb{E}\left(M^{2}\right)=\sum_{k=0}^{s-1} \sum_{m_{1}=0}^{s} \cdots \sum_{m_{\delta}=0}^{s} \pi\left(k, m_{1}, \ldots, m_{\delta}\right)(s-k)^{2} .
$$

In case $\delta=0$, we obviously have that $\mathbb{E} R=0$. To determine $\mathbb{E} R$ for $\delta=1$, note that $R$ satisfies

$R \stackrel{d}{=}\left(\sum_{j=1}^{\alpha+M_{1}} A_{1, j}-s\right)^{+}(s-X)^{+}$, where $\stackrel{d}{=}$ denotes equality in distribution, and hence

$$
\begin{aligned}
\mathbb{E} R & =\mathbb{E}\left(\sum_{j=1}^{\alpha+M_{1}} A_{1, j}-s\right)^{+}(s-X)^{+}, \\
& =\sum_{k=0}^{s-1} \sum_{m_{1}=0}^{s} \pi\left(k, m_{1}\right) \mathbb{E}\left(\sum_{j=1}^{\alpha+m_{1}} A_{1, j}-s\right)^{+}(s-k) .
\end{aligned}
$$

With $\max \{x, 0\}=x-\min \{x, 0\}$ we get

$$
\begin{aligned}
& \mathbb{E}\left(\sum_{j=1}^{\alpha+m_{1}} A_{1, j}-s\right)^{+} \\
& \quad=\left(\alpha+m_{1}\right) \mu_{A}-s-\sum_{n=0}^{s-1} a_{n}^{\alpha+m_{1}}(n-s) .
\end{aligned}
$$

Substituting (42) into (41) gives an expression for $\mathbb{E} R$ that consists of finitely many terms only. Similar expressions can be obtained for $\delta \geq 2$. For $\delta=2$ we have

$$
R \stackrel{d}{=}\left(\left(\sum_{j=1}^{\alpha+M_{2}} A_{2, j}-s\right)^{+}+\sum_{j=1}^{\alpha+M_{1}} A_{1, j}-s\right)^{+}(s-X)^{+} .
$$

Introducing the notation

$$
U_{n}=\sum_{j=1}^{\alpha+M_{n}} A_{n, j}-s,
$$

we can write (43) as

$$
\begin{aligned}
R & \stackrel{d}{=}\left(U_{2}^{+}+U_{1}\right)^{+}(s-X)^{+} \\
& =\max \left\{0, U_{1}, U_{1}+U_{2}\right\}(s-X)^{+} .
\end{aligned}
$$

In general, we find for $\delta \geq 1$ that

$$
\begin{aligned}
R \stackrel{d}{=} & \max \left\{0, U_{1}, U_{1}+U_{2}, \ldots,\right. \\
& \left.U_{1}+U_{2}+\cdots+U_{\delta}\right\}(s-X)^{+},
\end{aligned}
$$

and conditioning as in (41) leads to explicit expressions for $\mathbb{E} R$.

Table 1 displays values of $\mathbb{E} X, \mathbb{P}(X \leq s-1), \mathbb{E}\left(M^{2}\right)$ and $\mathbb{E} R$ for $\eta=10$ and $A$ Poisson distributed with $\mu_{A}=0.99$. These values were obtained by determining the $s(s+1)^{\delta}$ boundary probabilities as outlined in Sect. 2. Observe that $\mathbb{E} X, \mathbb{E}\left(M^{2}\right)$ and $\mathbb{E} R$ increase as functions of $\delta$, and $\mathbb{E} X$ increases as a function of $\alpha$. The case $\alpha=5$ is extreme because the stability condition (3) is only just satisfied. $\mathbb{E} X$ becomes large because of the $\frac{1}{2} \alpha \sigma_{A}^{2} /\left(s-\alpha \mu_{A}\right)$ term, while $\mathbb{E}\left(M^{2}\right)$ and $\mathbb{E} R$ become negligibly small (mainly because $\mathbb{P}(X \leq s-1)$ is small $)$. 
Table $1 \mathbb{E} X, \mathbb{P}(X \leq s-1), \mathbb{E}\left(M^{2}\right), \mathbb{E} R$ for $\eta=10$ and $A$ Poisson distributed with $\mu_{A}=0.99$

\begin{tabular}{|c|c|c|c|c|c|c|}
\hline \multirow[t]{2}{*}{$\alpha$} & \multicolumn{3}{|l|}{$\mathbb{E} X$} & \multicolumn{3}{|c|}{$\mathbb{P}(X \leq s-1)$} \\
\hline & $\delta=0$ & $\delta=1$ & $\delta=2$ & $\delta=0$ & $\delta=1$ & $\delta=2$ \\
\hline 0 & 5.2133 & 5.3684 & 5.4777 & 0.8625 & 0.8164 & 0.7939 \\
\hline 1 & 5.2759 & 5.4614 & 5.5911 & 0.8282 & 0.7785 & 0.7555 \\
\hline 2 & 5.3791 & 5.6076 & 5.7653 & 0.7713 & 0.7213 & 0.6999 \\
\hline 3 & 5.5830 & 5.8781 & 6.0783 & 0.6637 & 0.6230 & 0.6067 \\
\hline 4 & 6.1847 & 6.5984 & 6.8776 & 0.4447 & 0.4229 & 0.4150 \\
\hline 5 & 54.7128 & 55.3828 & 55.8741 & 0.0145 & 0.0140 & 0.0139 \\
\hline \multirow[t]{2}{*}{$\alpha$} & \multicolumn{3}{|l|}{$\mathbb{E}\left(M^{2}\right)$} & \multicolumn{3}{|l|}{$\mathbb{E} R$} \\
\hline & $\delta=0$ & $\delta=1$ & $\delta=2$ & $\delta=0$ & $\delta=1$ & $\delta=2$ \\
\hline 0 & 35.6196 & 38.2757 & 39.3444 & 0 & 1.5931 & 2.7066 \\
\hline 1 & 23.8530 & 25.6764 & 26.3761 & 0 & 1.5184 & 2.5753 \\
\hline 2 & 14.6221 & 15.6768 & 16.0687 & 0 & 1.4003 & 2.3640 \\
\hline 3 & 7.7711 & 8.2424 & 8.4145 & 0 & 1.2060 & 2.0219 \\
\hline 4 & 3.0273 & 3.1733 & 3.2232 & 0 & 0.8540 & 1.4299 \\
\hline 5 & 0.0552 & 0.0573 & 0.0579 & 0 & 0.0336 & 0.0584 \\
\hline
\end{tabular}

The entries in Table 1 for $\alpha=0$ (so $s=10$ ) and $\delta=2$ require the determination of 1210 boundary probabilities, and hence solving a system of equally many linear equations. For even larger values of $s$ and $\delta$ the set of boundary probabilities may become prohibitively large. It is therefore that we search for approximations for $\mathbb{E}\left(M^{2}\right)$ and $\mathbb{E} R$ that can be easily calculated for larger values of $s$ and $\delta . \mathbb{E}\left(M^{2}\right)$ can be written as $\mathbb{E}\left(M^{2}\right)=\sum_{j=0}^{s-1} \mathbb{P}(X=j)(s-j)^{2}$, from which we find that

$$
\begin{aligned}
\left(\sum_{j=0}^{s-1} \mathbb{P}(X=j)(s-j)\right)^{2} & \leq \sum_{j=0}^{s-1} \mathbb{P}(X=j)(s-j)^{2} \\
& \leq s \sum_{j=0}^{s-1} \mathbb{P}(X=j)(s-j)
\end{aligned}
$$

where the first inequality follows from Jensen's inequality. Together with (33) this leads to the bounds

$\left(\frac{s-\alpha \mu_{A}}{1+\mu_{A}}\right)^{2} \leq \mathbb{E}\left(M^{2}\right) \leq s \frac{s-\alpha \mu_{A}}{1+\mu_{A}}$.

These bounds, and some further improvements, have been presented in [4]. No simple bounds on $\mathbb{E} R$ can be derived. Instead, we derive a fluid approximation for $\mathbb{E} R$ in the next subsection.

\section{A fluid approximation}

We now employ a heuristic argument to construct an approximation for $\mathbb{E} R$, which, together with the bounds (48), gives an approximation for $\mathbb{E} X$. The argument is based on inspection of the sample paths of various realizations of the process defined by (1).

The cyclic behavior discussed in Sect. 1.2 is typically observed in case $\mu_{A}>1$ and $\delta \geq 1$, irrespective of the actual distribution of $A$, suggesting that we can construct a deterministic approximation of the sample path. Our approximation for $\mathbb{E} R$ is then obtained by evaluating $\mathbb{E} R$ for this deterministic approximation.

More formally, we define a deterministic process $\left\{x_{n}\right\}$ from (1) by replacing $A_{n}$ by its expected value $\mu_{A}$, yielding

$x_{n+1}=\left(x_{n}-s\right)^{+}+\mu_{A}\left(\alpha+\left(s-x_{n-\delta}\right)^{+}\right)$.

Given initial values $x_{0}=\cdots=x_{\delta}=\alpha \mu_{A}$, it is easy to see that (49) yields for $j=1, \ldots, \delta+1$

$x_{\delta+j}=j\left(\eta-\alpha \mu_{A}\right) \mu_{A}-(j-1) s$.

At the beginning of slot $2 \delta+1$ the queue has built up to the level $(\delta+1)\left(\eta-\alpha \mu_{A}\right) \mu_{A}-s \delta$, after which the queue is drained at rate $s-\alpha \mu_{A}$. This yields

$x_{2 \delta+1+j}=(\delta+1)\left(\eta-\alpha \mu_{A}\right) \mu_{A}-(\delta+j) s+j \alpha \mu_{A}$,

for $j=1, \ldots, L^{*}$. Here $L^{*}$ is the smallest value $l$ for which $x_{2 \delta+1+l}$ hits $\alpha \mu_{A}$. Consequently, an approximation for $L^{*}$ can be obtained from $x_{2 \delta+1+L^{*}} \approx \alpha \mu_{A}$, that is,

$L^{*} \approx \frac{(\delta+1)\left(\eta-\alpha \mu_{A}\right) \mu_{A}-s \delta}{s-\alpha \mu_{A}}$. 
After instant $2 \delta+1+L^{*}$ the sequence repeats itself. Hence the cycle length equals $L=2 \delta+1+L^{*} \approx(\delta+1)\left(\mu_{A}+1\right)$. We therefore approximate $\mathbb{E} R$ as

$$
\begin{aligned}
\mathbb{E} R & \approx \lim _{N \rightarrow \infty} \frac{1}{N} \sum_{n=1}^{N}\left(s-x_{n}\right)^{+}\left(x_{n+\delta}-s\right)^{+} \\
& \approx \frac{1}{L} \sum_{n=1}^{L}\left(s-x_{n}\right)^{+}\left(x_{n+\delta}-s\right)^{+} .
\end{aligned}
$$

For $\mu_{A}>1$, we can approximate the latter sum by the terms $n=2, \ldots, \delta+1$ (since $s-x_{n}>0$ ), so that

$$
\begin{aligned}
\mathbb{E} R \approx & \frac{1}{L} \sum_{j=1}^{\delta}\left(s-\alpha \mu_{A}\right)^{+} \\
& \times\left(j\left(\eta-\alpha \mu_{A}\right) \mu_{A}-(j-1) s-s\right)^{+} \\
= & \frac{1}{L} \frac{1}{2} \delta(\delta+1)\left(s-\alpha \mu_{A}\right)\left(\left(\eta-\alpha \mu_{A}\right) \mu_{A}-s\right)^{+} \\
\approx & \frac{1}{2} \frac{\delta}{\mu_{A}+1}\left(s-\alpha \mu_{A}\right)\left(\left(\eta-\alpha \mu_{A}\right) \mu_{A}-s\right)^{+} .
\end{aligned}
$$

Substituting (52) into (30) yields the following approximation for $\mathbb{E} X$.

$$
\begin{aligned}
\mathbb{E} X \approx & \frac{\alpha \sigma_{A}^{2}}{2\left(s-\alpha \mu_{A}\right)}+\frac{\sigma_{A}^{2}}{2\left(1+\mu_{A}\right)} \\
& +\frac{s+\alpha \mu_{A}}{2}+\mathbb{E}\left(M^{2}\right) \frac{\mu_{A}^{2}-1}{2\left(s-\alpha \mu_{A}\right)} \\
& +\frac{1}{2} \delta \frac{\mu_{A}}{\mu_{A}+1}\left(\left(\eta-\alpha \mu_{A}\right) \mu_{A}-s\right)^{+} .
\end{aligned}
$$

The bounds in (48) for $\mathbb{E}\left(M^{2}\right)$ can again be used to obtain explicit expressions.

In order to assess the quality of (53) we have carried out a number of simulations. Denote by $\alpha^{*}$ the mean acquisition effort per slot, i.e.,

$$
\alpha^{*}=\frac{\eta}{1+\mu_{A}} \text {. }
$$

The mean number of acquired customers per slot, denoted by $\lambda$, then equals $\alpha^{*} \mu_{A}$.

Figures 6 and 7 display $\mathbb{E} X$ (obtained by simulation) as a function of $\lambda$ for $A$ Poisson, $\eta=10, \alpha=0$ and $\delta=1$, 5,10 . The results we present are based on a simulation of $10,000,000$ slots, in which we start from an empty queue and neglect the first 1,000,000 slots. The dashed lines represent the approximations that follow from (53) (where we substitute for $\mathbb{E} M^{2}$ the average of the two bounds in (48)). Figure 6 leads us to conclude that the approximations are excellent for $\alpha=0$. There are some marked differences between Figs. 6 and 7. Most importantly, the approximation

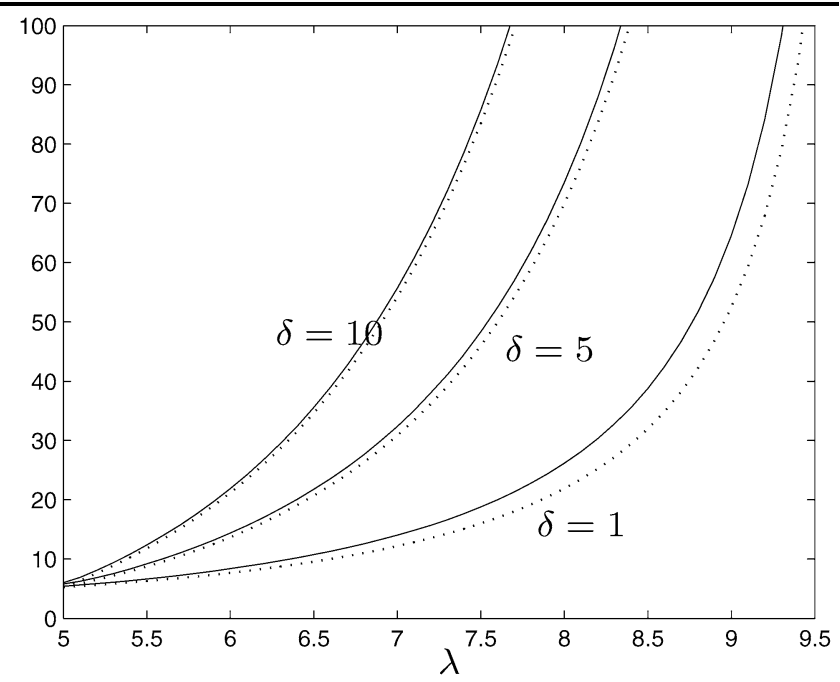

Fig. $6 \mathbb{E} X$ for $A$ Poisson distributed with $\mu_{A}=3, \eta=10, \alpha=0$ and $\delta=1,5,10$. The dashed lines represent the approximations that follow from (53)

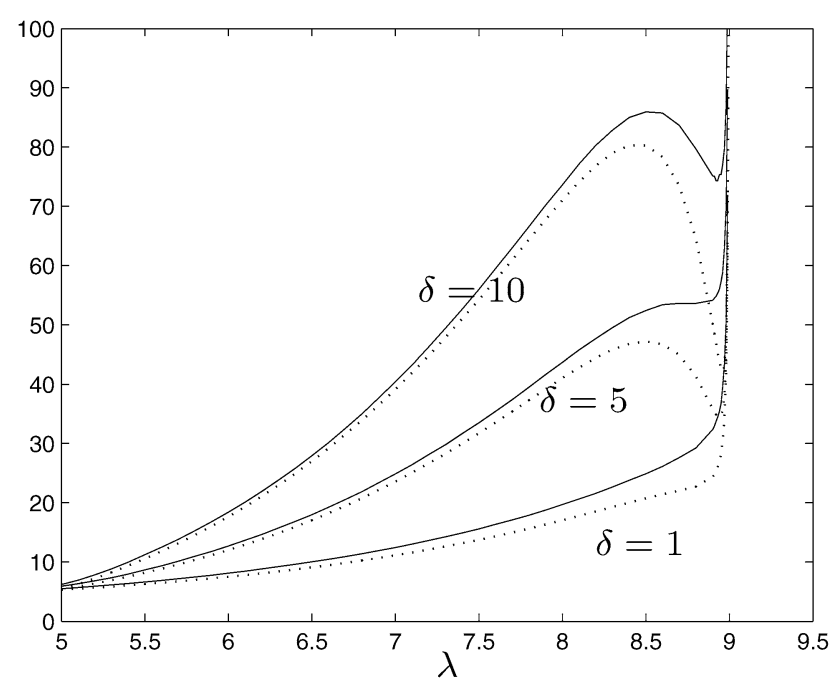

Fig. $7 \mathbb{E} X$ for $A$ Poisson distributed with $\mu_{A}=3, \eta=10, \alpha=1$ and $\delta=1,5,10$. The dashed lines represent the approximations that follow from (53)

(53) is less accurate in case $\alpha=1$. This is so in particular for the higher traffic intensities.

From these and many other examples we conclude that (53) is in general sharp, but breaks down in heavy traffic conditions for $\alpha \geq 1$. The latter is because the deterministic approximation to the sample path is less convincing for increasing values of $\alpha$, compare e.g. Figs. 1-3. Also, obviously, the deterministic approximation of the sample path is more convincing for large values of $\delta$.

4.1 Properties of the mean queue length

Approximation (53) suggests various interesting properties for $\mathbb{E} X$. Firstly, consider $\mathbb{E} X$ as a function of the delay $\delta$. 
Equation (53) suggests that $\delta$ has no impact on the mean queue length in case $\mu_{A} \leq 1$. However, in case $\mu_{A}>1, \mathbb{E} X$ increases linearly with $\delta$. It follows in particular that the correlation term $\mathbb{E} R$ is the dominating term in the expression for $\mathbb{E} X$, and that $\mathbb{E} X$ grows without bounds for $\delta$ tending to infinity.

Secondly, consider $\mathbb{E} X$ as function of $\alpha$. In order to set $\alpha$ such that the mean limiting queue length is kept small, there are two considerations. The smaller $\alpha$, the quicker the queue is emptied, while the larger $\alpha$, the more the arrival process is smoothened. The approximation (53) can be used to strike the proper balance between these two considerations.

Thirdly, consider $\mathbb{E} X$ as function of $\lambda$. Equation (53) suggests that the mean limiting queue length is not necessarily monotonic in the traffic intensity for $\delta \geq 1$ and $\alpha \geq 1$ (see also Fig. 7). To see this, observe that the approximation (52) of $\mathbb{E} R$ is not monotonic in $\mu_{A}$. This follows easily as this approximation is nonnegative, and equals 0 both for small values of $\mu_{A}$ (i.e. $\left.\mu_{A} \leq 1\right)$ and for $\mu_{A}=s / \alpha$. Now for large values of $\delta$, the correlation term will dominate $\mathbb{E} X$, which then is non-monotonic too.

This non-monotonicity can be explained informally as follows. Observe that the input to the queue consists of two sources: $\left(X_{n}-s\right)^{+}$and a sum which increases in $\left(s-X_{n-\delta}\right)^{+}$. As the traffic intensity approaches the stability bound, the cyclic behavior of the sample paths vanishes. Hence, increasing the traffic intensity causes the input to be less bursty, and this results in smaller queue lengths. Another way to see this is by observing that the bursts of arriving customers that follows periods in which the system is (relatively) empty are caused by an inflow of magnitude $\left(s-\alpha \mu_{A}\right) \mu_{A}+\alpha \mu_{A}=\left(\eta-\alpha \mu_{A}\right) \mu_{A}$. The latter expression is non-monotonic in $\mu_{A}$. This type of non-monotonic behavior, though remarkable, is not uncommon in systems that involve control and feedback delay. Situations in which these characteristics lead to unwanted oscillations and increased delay occur if the traffic dynamics can be expressed via a difference equation or differential equation that involves a delayed response, see e.g. [5-7].

\section{Free acquisition planning}

We have discussed in Sect. 1.2 how the delay $\delta$ may result in cyclic behavior and a strongly correlated arrival process. This can have severe consequences for the mean queue length, see (30), since the correlation term $\mathbb{E} R$ becomes dominant in high-load situations. We now aim at smoothening the arrival process and reducing the correlation of the arrival process. We will do so by choosing an appropriate scheme for free acquisition planning, see (2).

Recall where the cyclic behavior comes from. More acquisition is performed when the queue is small, and less acquisition is performed when the queue is large. This type of acquisition planning is expected to lead to a smoother arrival process of customers. However, the delay $\delta$ upsets the balance. The impact of a corrective decision, like more acquisition if the system is less busy, is only seen $\delta$ slots later. If the system is busier $\delta$ slots later, the extra arrivals might just have the opposite effect. This phenomenon of control decisions that have the opposite effect as one had in mind is precisely what is captured by the correlation term. That is, $\mathbb{E} R=\lim _{n \rightarrow \infty} \mathbb{E}\left[\left(X_{n}-s\right)^{+}\left(s-X_{n-\delta}\right)^{+}\right]$might be viewed as a measure for the performance of the acquisition planning. Ideally, $\mathbb{E} R$ equals zero, and a high value of $\mathbb{E} R$ indicates that the type of acquisition planning balances the input poorly. It might be clear that the larger $\delta$, the more unlikely it is that the static acquisition planning adopted by the delayed acquisition queue balances the input well.

Our primary goal is to reduce the mean limiting queue length by constructing an acquisition scheme that balances the input properly despite a substantial delay. In balancing the input, one would want $\lambda$ customers to arrive to the queue each slot. This is not feasible, since we are dealing with a stochastic arrival process, but it might serve as a guiding principle. Say we are at the beginning of slot $n$. We have spent $\bar{\alpha}_{n-\delta}+\bar{\alpha}_{n-\delta+1}+\cdots+\bar{\alpha}_{n-1}$ units of energy on acquisition in the previous $\delta$ slots and we are still free to choose $\bar{\alpha}_{n}$, which makes that the number of arriving customers in the next $\delta+1$ slots is given by

$\sum_{k=0}^{\delta} \sum_{i=1}^{\bar{\alpha}_{n-k}} A_{k, i}$

where the $A_{k, i}$ are i.i.d. as $A$. Ideally, there will be $\eta-\bar{\alpha}_{n}$ customers at the beginning of slot $n$, so that in each slot all waiting customers can be served. In that case, we would have $X_{n}=\eta-\bar{\alpha}_{n}$ and

$\sum_{k=0}^{\delta} \sum_{i=1}^{\bar{\alpha}_{n-k}} A_{k, i}=(\delta+1) \lambda$.

In reality, this will not be the case, but we will take these values as a benchmark. So, we aim at choosing $\bar{\alpha}_{n}$ such that

$X_{n}-\left(\eta-\bar{\alpha}_{n}\right)+\sum_{k=0}^{\delta} \sum_{i=1}^{\bar{\alpha}_{n-k}} A_{k, i}=(\delta+1) \lambda$.

This benchmark gives rise to a useful scheme for acquisition planning. However, since we do not know the outcome of the $A_{k, i}$ in (57) we replace these random variables by their expectation $\mu_{A}$, and upon some rewriting we get

$$
\begin{aligned}
\bar{\alpha}_{n}^{*} & =\frac{1}{1+\mu_{A}}\left[\eta+(\delta+1) \lambda-X_{n}-\mu_{A} \sum_{k=1}^{\delta} \bar{\alpha}_{n-k}\right] \\
& =\alpha^{*}+\frac{1}{1+\mu_{A}}\left[(\delta+1) \lambda-X_{n}-\mu_{A} \sum_{k=1}^{\delta} \bar{\alpha}_{n-k}\right] .
\end{aligned}
$$




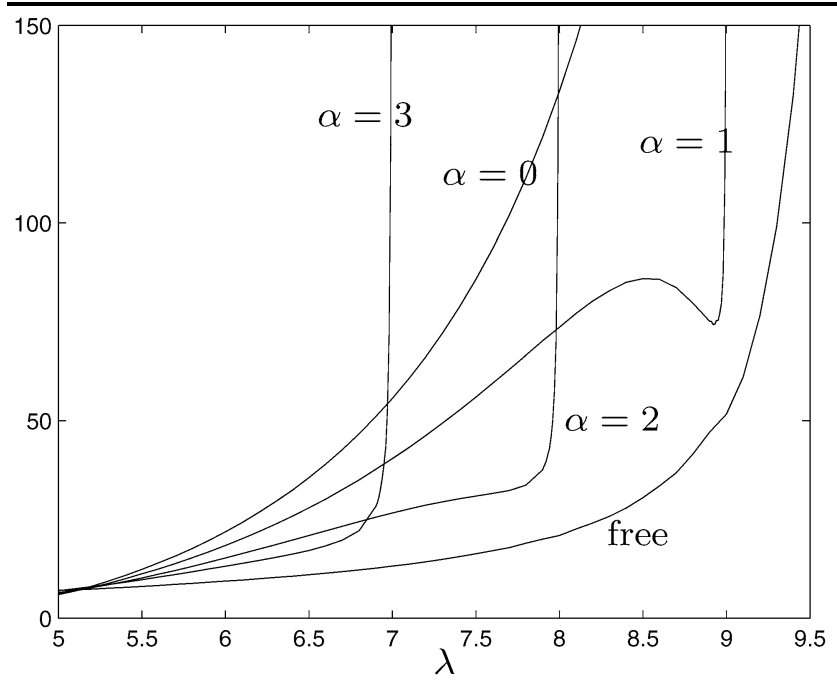

Fig. $8 \mathbb{E} X$ for $A$ Poisson distributed with $\eta=10, \delta=10$, for $\alpha=0$, $1,2,3$ and free acquisition planning (59)

To make sure that $\bar{\alpha}_{n}$ is integer-valued, and no energy is wasted, we then choose $\bar{\alpha}_{n}$ according to

$\bar{\alpha}_{n}=\max \left\{0,\left\lfloor\bar{\alpha}_{n}^{*}\right\rfloor, \eta-X_{n}\right\}$.

\section{Comparison of acquisition schemes}

In order to assess the merit of various acquisition schemes, we have carried out a number of simulations. We let $A$ be Poisson distributed, and set $\eta=10, \delta=10$. The results we present are based on a simulation of $10,000,000$ slots, in which we start from an empty queue and neglect the first $1,000,000$ slots. We also verify some of the properties that were stated in Sect. 4.1.

Figures 8 and 9 give results for static acquisition planning with $\alpha=0,1,2,3$ and free acquisition planning according to (59). Figure 8 displays the mean limiting queue length. The curves obtained from static acquisition planning all have an asymptote at $\lambda=\eta-\alpha$. For most values of $\lambda, \alpha=0$ results in the largest mean queue length, while (59) results in the smallest mean queue length. For static acquisition planning, the non-monotonic behavior mentioned in Sect. 4.1 is clearly visible for $\alpha=1$. Figure 9 displays the correlation term $\mathbb{E} R$. For static acquisition planning, $\mathbb{E} R$ tends to zero as $\lambda$ goes to its maximum sustainable value. Free acquisition planning succeeds in keeping the $\mathbb{E} R$ small, except for high values of $\lambda$, where the difference with static acquisition planning, case $\alpha=0$, is negligible. As mentioned before, a small correlation term indicates that the acquisition scheme balances the input well.

From many other simulations we observed that free acquisition performs better for increasing values of $\delta$. The

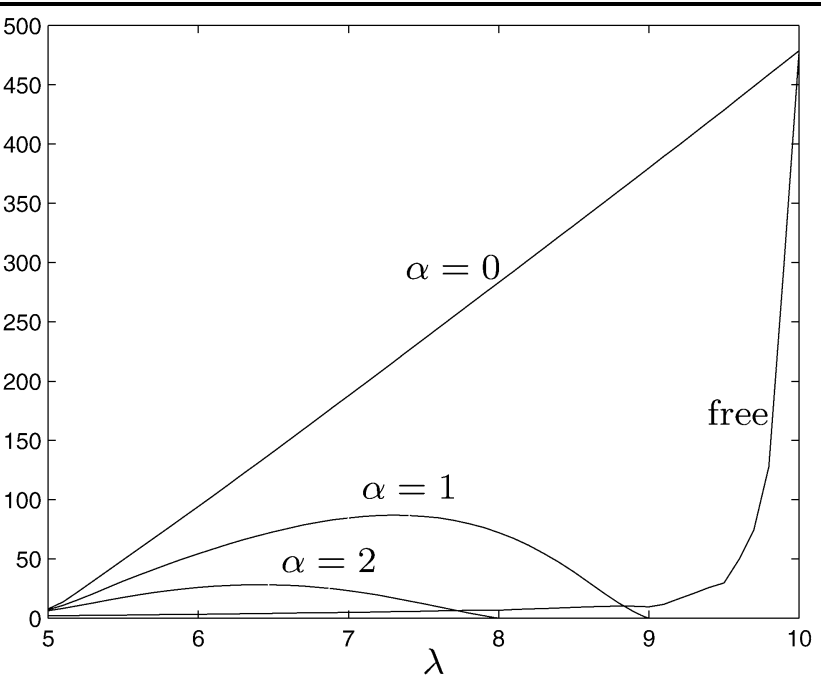

Fig. $9 \mathbb{E} R$ for $A$ Poisson distributed with $\eta=10, \delta=10$, for $\alpha=0$, $1,2,3$ and free acquisition planning (59)

larger $\delta$, the more (relatively) $\mathbb{E} R$ is reduced by free acquisition planning. This can be explained as follows. Equation (59) determines the appropriate acquisition effort by estimating the number of customers that will arrive in the future. Denote the total acquisition effort in the $\delta$ previous slots by $t$. The estimated number of future arrivals is then $t \mu_{A}$. Hence, the larger $\delta$, the larger $t$, and the more precise the estimation of the number of future arrivals will be. A similar argument can be used for describing the influence of the arrival distribution. The more volatile the distribution, the less accurate the estimation of the future arrivals.

\section{Conclusions}

We have introduced the acquisition queue as a new type of queueing model, and obtained the pgf of the limiting queue length distribution. Using a method of Kingman [8] and a fluid approximation, we have obtained an approximation to the mean limiting queue length. Simulations have shown that the approximation is precise, although it breaks down in heavy-traffic situations.

Both the exact solution and the approximation revealed a number of interesting properties of the acquisition queue. We showed that the mean limiting queue length is increasing in the delay $\delta$ and grows without limits for $\delta$ tending to infinity. Also, rather remarkably, we showed that for $\delta \geq 1$ and $\alpha \geq 1$, the mean limiting queue length is not monotonic in the traffic intensity. The mean limiting queue length first grows with the traffic intensity, but as the traffic intensity approaches its maximum sustainable value, the mean limiting queue length decreases substantially.

We proposed a type of acquisition planning that determines the acquisition effort in slot $n$ based on the queue 
length at the beginning of slot $n$ and total acquisition effort in the $\delta$ previous slots. Numerical examples showed good performance, mainly due to the fact that the correlation of the input process is reduced.

\section{References}

1. Adan, I.J.B.F., van Leeuwaarden, J.S.H., Winands, E.M.M.: On the application of Rouché's theorem in queueing theory. Oper. Res. Lett. 34, 355-360 (2006)

2. Denteneer, D., van Leeuwaarden, J.S.H.: The delayed bulk service queue: a model for a reservation process. In: Liang, X., Xin, Z., Iversen, V.B., Kuo, G.S. (eds.) Performance Challenges for Efficient Next Generation Networks, Proceedings ITC 19, pp. 909 918 (2005)

3. Denteneer, D., van Leeuwaarden, J.S.H., Resing, J.: Bounds for a discrete-time multi-server queue with an application to cable networks. In: Charzinski, J., Lehnert, R., Tran-Gia, P. (eds.) Providing
Quality of Service in Heterogeneous Environments, Proceedings ITC 18, pp. 601-610 (2003)

4. Denteneer, D., Janssen, A.J.E.M., van Leeuwaarden, J.S.H.: Moments series inequalities for the discrete-time bulk service queue. Math. Meth. Oper. Res. 61, 85-108 (2005)

5. Fendick, K.W., Rodrigues, M.A.: Asymptotic analysis of adaptive rate control for diverse sources with delayed feedback. IEEE Trans. Inform. Theory 40(6), 2008-2025 (1994)

6. Gyori, I., Ladas, G.: Oscillation Theory of Delay Differential Equations, with Applications. Clarendon Press, Oxford (1991)

7. Johari, R., Tan, D.K.H.: A new feedback congestion control policy for long propagation delays. IEEE/ACM Trans. Netw. 9, 818-832 (2001)

8. Kingman, J.F.C.: Inequalities in the theory of queues. J. Roy. Stat. Soc. Ser. B 32, 102-110 (1970)

9. van Leeuwaarden, J., Denteneer, D., Resing, J.: A discrete-time queueing model with periodically scheduled arrival and departure slots. Perform. Eval. 63, 278-294 (2006)

10. Neuts, M.F.: Structured Stochastic Matrices of M/G/1 Type and Their Applications. Dekker, Basel (1989) 